\title{
A New Procedure for Damage Assessment of Prestressed Concrete Beams Using Artificial Neural Network
}

\author{
K. Sumangala and C. Antony Jeyasehar \\ Department of Civil and Structural Engineering, Annamalai University, Tamilnadu, Annamalainagar 608 002, India \\ Correspondence should be addressed to K. Sumangala, josuma@rediffmail.com
}

Received 31 May 2011; Revised 24 August 2011; Accepted 24 August 2011

Academic Editor: Wilson Wang

Copyright (C) 2011 K. Sumangala and C. Antony Jeyasehar. This is an open access article distributed under the Creative Commons Attribution License, which permits unrestricted use, distribution, and reproduction in any medium, provided the original work is properly cited.

\begin{abstract}
A damage assessment procedure has been developed using artificial neural network (ANN) for prestressed concrete beams. The methodology had been formulated using the results obtained from an experimental study conducted in the laboratory. Prestressed concrete (PSC) rectangular beams were cast, and pitting corrosion was introduced in the prestressing wires and was allowed to be snapped using accelerated corrosion process. Both static and dynamic tests were conducted to study the behaviour of perfect and damaged beams. The measured output from both static and dynamic tests was taken as input to train the neural network. Back propagation network was chosen for this purpose, which was written using the programming package MATLAB. The trained network was tested using separate test data obtained from the tests. A damage assessment procedure was developed using the trained network, it was validated using the data available in literature, and the outcome is presented in this paper.
\end{abstract}

\section{Introduction}

Concrete structures are designed and constructed to suit the requirements of its time. Damages may be unavoidable during its design life time due to various reasons. A structure which is said to be well designed may survive the damage but safety is not guaranteed. When the damage goes undetected and unrepaired, it will lead to failure or may demand costly repair and huge loss of life. Therefore, the problem of maintenance and repair of existing structures involves damage detection at an early stage. For massive structures like bridges which were constructed some 50 to 60 years ago, it is necessary to test their functionality under the present loading condition and quantify damage, if any, since demolishing and reconstructing them would involve huge expenditures. Evaluating the residual life and remaining load-carrying capacity of these structures is also important. Damage can be defined as the change in performance of structures, which can be identified in terms of crack formation and a consequent stiffness reduction. Damage recognition and location are the key factors in the design of a structural health monitoring system. When damage lies inside the structure and is not visible to the naked eye, it is possible to locate and quantify the damage with nondestructive tests and various analytical models [1]. Vibration-based damage detection methods seem to be effective at detecting and localizing the damage on full-scale structures [2].

The easiest and simplest way to detect damage with dynamic parameters is by noting the changes in the natural frequency of the system. Cawley and Adams [3] formulated a scheme to detect damage in composite materials from natural frequency shifts. Abdel Wahab and Roeck [1] investigated the application of the change in modal curvatures to detect the damage in a prestressed concrete bridge. They modelled simply supported and continuous beams using finite elements and introduced damage at different locations in terms of reduced stiffness of the corresponding elements, and they concluded that the natural frequencies of the damaged and undamaged beams indicated the presence of damage in a global sense. Abdul Razak and Choi [4] studied the effect of general corrosion on the modal parameters of reinforced concrete beams. Modal tests were performed and compared against that from a control beam. The changes were inconsistent with the changes in natural frequencies, but a trend observed was mode dependent. The load carrying capacity of the beams was determined through static load test, and 
the results were correlated with the state of corrosion damage and changes in the modal parameters. From the changes in natural frequencies, stiffness degradation was estimated and compared.

Carpinteri et al. [5] have discussed some aspects of the NDE techniques in in situ damage assessment. They have taken up the study on assessing the stability of a historical masonry tower using the NDE technique called thermography and with nonlinear numerical simulations. According to the authors, the results of the analysis gave valuable hints about how much damage had occurred and when the restoration should take place.

Wu et al. [6] illustrated the neural network-based methodology to show that this approach could be used to identify member damage. The authors used the Fourier spectra of the computed relative acceleration, generated from a numerical model of a simple three-storey frame, as an input to neural network. According to their results, ANNs can learn about the behaviour of undamaged and damaged structures to identify the damaged member and the extent of damage from patterns in the frequency response of a structure.

According to Chen et al. [7], neural network can distinguish small differences between the transmissibility functions which carry the information of structural faults. The transmissibility function is sensitive to structural faults and easy to measure in the situations where the excitations of the structural system are not available or immeasurable. They had also suggested that transmissibility is a useful feature for training neural network as a fault diagnosis model. Fang et al. [8] referred the features of various training algorithms and with that as basis implemented the neural network to the frequency-response-functions- (FRFs-) based structural damage detection. The analysis results on a cantilever beam show that, in all considered damage cases (i.e., trained damage cases and unseen damage cases, single-damage cases and multiple-damage cases), the neural network can assess damage conditions with very good accuracy.

Zapico et al. [9] in their paper described a procedure for damage assessment in a two-storey steel frame and steel-concrete composite floors structure. The procedure is based on a multilayer perceptron (MLP). A simplified finite element model was used to generate the training data. This model was previously updated through another MLP using two natural frequencies as inputs and the stiffness of the beams and masses as updating parameters. The different combinations of damage at the ends of the longitudinal beams were used as damage scenarios. The training data for the MLP were generated by varying at random the stiffness of the longitudinal beams. Two natural frequencies and mode shapes were used as inputs, and three different definitions of damage (sections, bars, and floors) were tried as outputs. MLPs were trained through the error back-propagation algorithm. Finally, the performance of the procedure was evaluated through the experimental data. Only the approach of damage at floor level gives reasonable results.

Yuen and Lam [10] had reviewed the works of many researchers on damage detection using ANN. They have also developed an ANN based design method which does not need user's judgment. By using the proposed ANN-design method both location, and severity of damage could be known. The method was employed in an example to demonstrate their proposed methodology. Norhisham Bakhary et al. [11] in this study proposed a statistical approach to take into account the effect of uncertainties in developing an ANN model. By applying Rosenblueth's point estimate method verified by Monte Carlo simulation, the statistics of the stiffness parameters were estimated. The probability of damage existence (PDE) is then calculated based on the probability density function of the existence of undamaged and damaged states. The developed approach is applied to detect simulated damage in a numerical steel portal frame model and also in a laboratory-tested concrete slab. The effects of using different severity levels and noise levels on the damage detection results were discussed.

de Lautour and Omenzetter [12] in this study present a general method for predicting seismic-induced damage using artificial neural networks (ANNs). The approach was to describe both the structure and ground motion using a large number of structural and ground motion properties. The class of structures analysed were 2D reinforced concrete (RC) frames that varied in topology, stiffness, strength, and damping and were subjected to a suite of ground motions. Dynamic structural responses were simulated using nonlinear FEM analysis and damage indices describing the extent of damage calculated. Using the results of the numerical simulations, a mapping between the structural and ground motion properties and the damage indices was than established using an ANN. The performance of the ANN was assessed using several examples, and the ANN was found to be capable of successfully predicting damage.

Though more works have been done involving reinforced concrete and steel structures using ANN, not much work has been done on PSC members. Modelling of damaged prestressed concrete beam is complicated, and usage of conventional methods for damage assessment of Prestressed Concrete (PSC) beams thus becomes difficult. ANN is a possible solution in this situation. Therefore, a well-designed neural network is able to serve as a real time data processor for damage assessment.

The main focus of this work was to train and test the network only with natural frequency and stiffness of damaged and perfect beams. Widely used feed forward ANN that learns with back propagation algorithm was adopted, and details about network architecture are presented. Further, it has been demonstrated that it is possible to predict the damage with acceptable accuracy by just feeding the current stiffness and natural frequency of the damaged structure [13] as inputs to the trained ANN. A novel in situ damage assessment procedure that needs only limited nondestructive measurements on the damaged structure is proposed.

The newness of this procedure is that it requires only minimum data collected from the damaged structure and theoretical data developed for the original structure. So, with minimum number of inputs, the procedure is adopted. No further experiments are needed to get data in order to adopt procedure. 


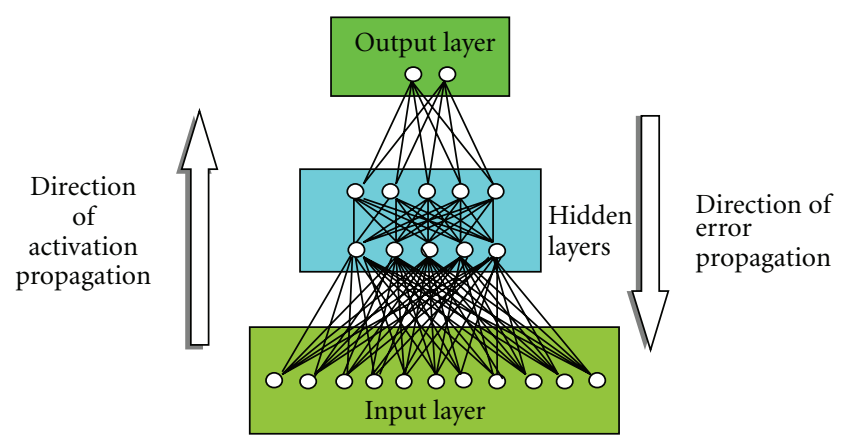

FIGURE 1: Architecture of the back propagation network.

\section{Feed Forward Artificial Neural Network and Back Propagation Algorithm}

Even though the ANN and back propagation algorithm are well known, this section briefly revisits the concepts of feed forward that learns by back propagation algorithm used in the damage assessment of prestressed concrete beams presented in this paper. The processing units in this artificial neural network are arranged in layers, that is, input layer, an output layer, and a number of hidden layers as shown in Figure 1. The hidden units presented here allow the network to represent and compute more complicated associations between patterns. The inputs are presented to the input layer. Each neuron in the hidden layer receives the weighted sum from the input layer and transfers its result to the output layer. The back propagation-learning algorithm calculates the error between the generated output and targeted output and uses the estimated error to modify the weight in response to the errors. The back propagation algorithm learns by changing its weights to follow the steepest path towards the bottom of a bowl shaped error surface. This process continues until a set of weights, which processes data accurately for the application, is found out. The final weights represent what the network has learnt and enable to infer for other data. Processing element of this network with specified inputs is shown in Figure 2.

Once the type of ANN and the learning algorithm are decided, it is necessary to generate the required training and test data. When the modeling of damaged PSC beam is difficult, one needs to depend upon the experimentally acquired data on the structure under study for training and testing the ANN. From theory, it is well known that the dynamic and/or static characteristics of the damaged PSC beams carry information about the damaged state of the structures. The ANN is made to recognize the damage occurred in the structure under study by presenting the measured dynamic and/or static data of the damaged and undamaged beams obtained experimentally. The following section presents the details of the experimental program carried out to generate the necessary training and test data for the ANN.

\section{Experimental Study}

Fivepost tensioned concrete rectangular beams were used in this investigation. The beams were of uniform cross section, that is, $125 \times 250 \times 4200 \mathrm{~mm}$. At each end, there were anchorage zones with enlarged section of size $350 \times 230 \times$ $250 \mathrm{~mm}$ and length of $230 \mathrm{~mm}$. The overall length of the beam was $4860 \mathrm{~mm}$, out of which $4590 \mathrm{~mm}$ was considered as the test zone. In order to achieve accessibility to prestressing tendons where the damage is introduced by corrosion, an opening $100 \times 120 \times 60 \mathrm{~mm}$ wide was provided in the middle bottom side of the beam. The beams were provided with nominal reinforcement in order to take up the handling stresses. The longitudinal reinforcement consists of two high yield strength deformed (HYSD) bars of $8 \mathrm{~mm}$ diameter at the top and the bottom. The stirrups were made using mild steel bars of $6 \mathrm{~mm}$ diameter and were provided with a spacing of $175 \mathrm{~mm}$. At the anchorage zone, 12 bars of $6 \mathrm{~mm}$ diameter were provided between 25 and $250 \mathrm{~mm}$ from the edge. Reinforcement details were kept the same for all the beams. The beams were cast with a mean concrete strength of $57 \mathrm{~N} / \mathrm{mm}^{2}$, posttensioned to a stress level of $0.7 f_{\mathrm{pu}}$. The ultimate strength of the high tensile steel (HTS) wire is $1715.2 \mathrm{~N} / \mathrm{mm}^{2}$. Six HTS wires of $7 \mathrm{~mm}$ diameter were used for each beam. The first beam, designated as $\mathrm{P7B1}$, was a perfect beam with absolutely no damage. The other four beams, namely, P7CB1, P7CB2, P7CB3, and P7CB4 were damaged to 33.33, 50, 66.67, and 83.33\%, respectively. The damage percentage represents the percentage of wires snapped. Snapping of wires was done using the accelerated electrochemical process. Through this process, pitting corrosion was induced and the wires were snapped. The setup made for inducing corrosion damage using accelerated electrochemical process is shown in Figure 3. After damaging the beams, static and dynamic tests were conducted on all five beams.

3.1. Testing of Beams. All the beams were subjected to both static and dynamic tests. The beams were tested under repeated loading at an interval of $10 \mathrm{kN}$ each (load stage). Four cycles of repeated load were applied in each load stage. The beams were repeatedly loaded till the failure load is reached. Deflections and strains were measured for a load increment of $2.5 \mathrm{kN}$ up to failure. Crack width of five cracks in the constant bending moment zone was also measured using a crack detection microscope of $0.02 \mathrm{~mm}$ precision. Static tests were conducted for determining the moment curvature, load deflection variations with loading in addition to the evaluation of ultimate load carrying capacity of the test beams.

Dynamic test was conducted at every $10 \mathrm{kN}$ load interval and after four cycles of load application at each stage. The dynamic response was picked up by linear variable differential transducer (LVDT) placed at mid span in the form of displacement history for the beams at all the chosen load stages. It was utilised for the frequency analysis. The time history records were analysed using the software DASYLAB for obtaining the natural frequencies. 


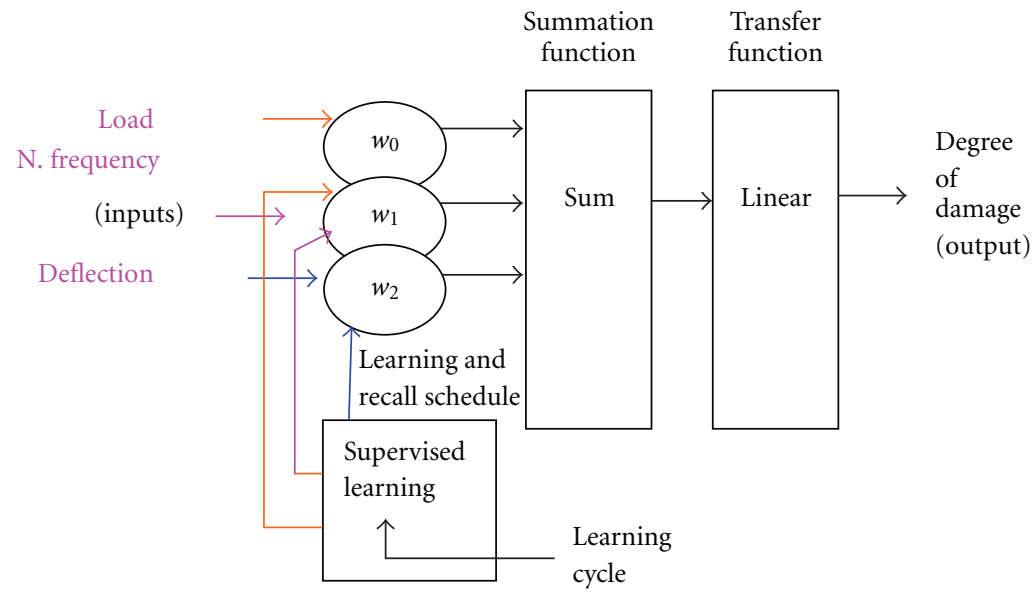

FIgURE 2: Processing element of this network with specified inputs.

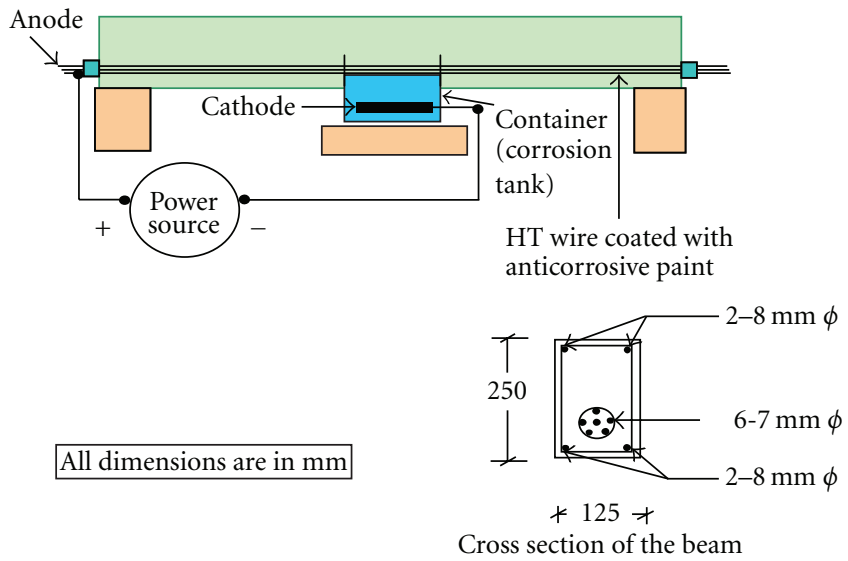

Figure 3: Corrosion induction setup.

\section{Generation of Training and Test Data for ANN and Its Architecture}

It was decided to use all the output obtained from the static and dynamic tests such as stiffness $(s)$, natural frequency $(f)$, deflection $(\delta)$, load at first crack $\left(P_{\mathrm{cr}}\right)$, crack width $(w)$, and ultimate load $\left(P_{u}\right)$ from the perfect and damaged beams for training the neural network. It may be difficult to assess the damage using the conventional methods since mathematical model is required to explain these behaviours. ANN does not require prior mathematical model to solve a problem which is a useful aspect of neural network.

4.1. Generation of Training and Test Data from Experiments. The applied load and the corresponding stiffness, natural frequency, deflection, and crack width values of damaged and undamaged beams (P7B1, P7CB1, P7CB3, and P7CB4) were obtained at regular intervals from the static and dynamic tests. Even though two sets of beams were tested and data collected for each case, only one data set was used for training. However, the ANN needs a larger volume of data so that it learns better and predicts the damage accurately.
ANN can be robust if large amount of training data is made available since training with large data can avoid the problem of over fitting and it can be fault tolerant with significant amount of redundancy and better learning algorithms. Over fitting or over learning is indicated by the inability of a network to perform better when unseen test data is presented to the network even though it is able to classify the training data. When large amount of data is used for training and the structure and size of the network are chosen properly to handle the data, the problem of over fitting can be avoided. To achieve this, the experimentally obtained results were linearly interpolated using the software ORIGIN to get the values at small regular intervals of load $(1 \mathrm{kN})$ thus making the training and test database larger.

The sample training data are given in Table 1. The data were normalized. The natural frequency, deflection, and crack width data obtained for the beam P7CB2 damaged to the extent of $50 \%$ in a similar manner were used as exclusive test data (Table 2). This enables us to test the ability of ANN to generalize when presented with totally unseen data. In addition to these test data, the static and dynamic data for $40 \%$ and $70 \%$ damage levels were also obtained by interpolation of the data acquired through experiments, and these data were also used as test data.

4.2. Architecture of the ANN. Feed forward neural network learning by back propagation (BP) algorithm written in MATLAB has been used, and its ability to predict damage just from the current natural frequency and postcrack stiffness obtained from the load-deflection characteristics of the damaged beam has been studied by training and testing the ANNs for various cases of input and comparing their performance for various input conditions. Postcrack stiffness of the damaged beam was also considered along with natural frequency since the frequency changes alone are insufficient to quantify the damage. To achieve this, the basic structure of the ANN was maintained and only the number of input nodes in the input layer was changed. During training process, different nodes were tried by trial and error for the hidden 
TABLe 1: Sample training data.

\begin{tabular}{lcccc}
\hline & & Natural frequency $(\mathrm{Hz})$ at various damage levels & \\
Load $\mathrm{kN}$ & $0 \%$ & $33.33 \%$ & $66.66 \%$ & $83 \%$ \\
& P7B1 & P7CB1 & 21.36 & 20.75 \\
\hline 0 & 23.5 & 22.58 & 21.36 & 20.75 \\
10 & 23.5 & 22.58 & 21.36 & 20.14 \\
20 & 23.5 & 22.58 & 20.75 & 18.92 \\
30 & 23.5 & 21.97 & 19.53 & - \\
40 & 22.28 & 21.36 & - \\
50 & 21.36 & 20.75 & - \\
\hline
\end{tabular}

TABle 2: Sample test data.

\begin{tabular}{lc}
\hline Load $\mathrm{kN}$ & Natural frequency $(\mathrm{Hz})$ at $50 \%$ damage $(\mathrm{P} 7 \mathrm{CB} 2)$ \\
\hline 0 & 21.36 \\
10 & 21.36 \\
20 & 21.36 \\
30 & 20.75 \\
40 & 20.14 \\
50 & 19.53 \\
\hline
\end{tabular}

layers to achieve the given performance accuracy, which is user defined. After various trials, these nodes were finalized, which gave the optimum output. So these numbers of nodes were chosen based on the performance accuracy requirement. The specifications of the ANN that gave the best performance and are used in this study are as follows.

(i) Number of input nodes in the input layer: $2-5$ for different cases.

(ii) Number of output nodes in the output layer: 1 .

(iii) Number of hidden layers: 2 with 7 and 5 nodes, respectively.

(iv) Training algorithm used: back propagation.

(v) Learning method: supervised learning.

(vi) The output of the neural network is the predicted extent of damage in the beam.

\section{ANN Training and Testing}

This section presents the results of ANN training and test details for different cases and analyses the suitability of ANN for damage assessment of PSC beams. Mainly, the focus of the study was to evaluate the effectiveness of ANN for damage assessment when trained only with natural frequency and stiffness of the damaged beam and with the mix of static and dynamic test data. Keeping this purpose in mind, the training of the ANN has been carried out for a changing number of inputs. However, the structure of the ANN and the training algorithm used were maintained as explained in the previous section for all the cases. In order to adopt the damage assessment procedure for various field beams having different cross sections, all the data were normalized before feeding into the network. The following were the cases studied in this work.

- Case i. ANN with applied load and natural frequency as input.

- Case ii. ANN with postcrack stiffness and natural frequency as input.

- Case iii. ANN with natural frequency (postcracking preyielding) as input.

- Case iv. ANN with applied load, natural frequency, and deflection as input.

- Case v. ANN with applied load, natural frequency, and crack width as input.

- Case vi. ANN with applied load, natural frequency, deflection, and crack width as input.

- Case vii. ANN with applied load, natural frequency, deflection, crack width, and ultimate load as input.

For the sake of practical application, only the first three cases are discussed in this paper. The remaining cases are explained elsewhere [14].

Case $i$ (ANN with applied load and natural frequency as inputs). This ANN is trained with applied load and natural frequencies obtained from the dynamic tests and aims at predicting the damage level from the average of possible solutions returned by the ANN for various test input data. The results obtained in this case are represented in a graphical form in Figure 4, which seems to be promising in the damage assessment process.

Case ii (ANN with stiffness and natural frequency as inputs). Each training or test data has two inputs, that is, stiffness (postcracking-preyielding) and natural frequency. This ANN has been studied so that a damage assessment procedure can be evolved that is suitable in practical situation. In a field beam, which is in the postcracking-preyielding stage, it is possible to obtain its current stiffness from its load deflection characteristics measured over a portion of the service load. A dynamic test on the same beam can return its natural frequency value. Therefore, these two parameters were selected for the ANN study and the network was trained, 

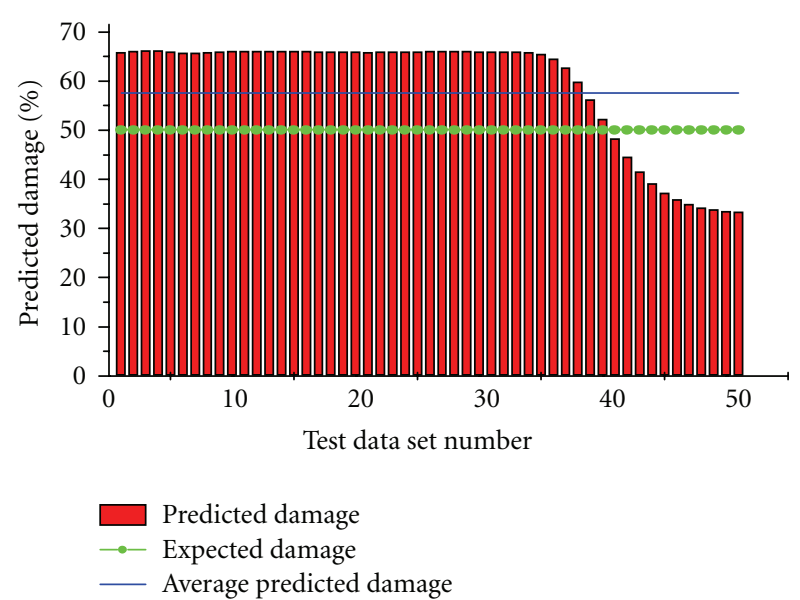

FIgURE 4: Input data set number versus damage predicted by ANN (Case i).

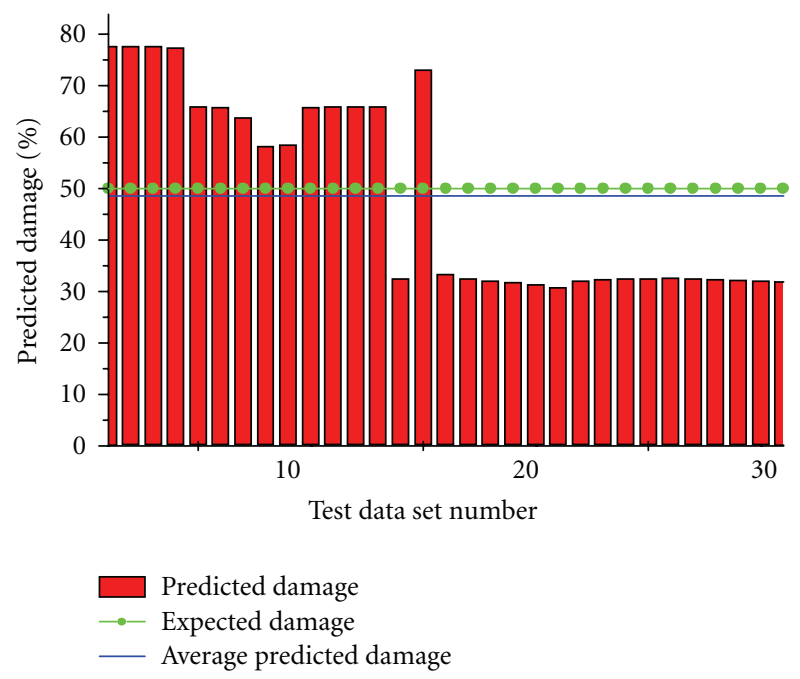

FIgURe 5: Input data set number versus damage predicted by ANN (Case ii).

and the result is presented below in the form of graph and is shown in Figure 5. However, the average of the predicted damage levels for different test data could closely predict the damage.

Case iii (ANN with natural frequency (postcracking-preyielding) as input). Each training or test data has only one input, that is, natural frequency. This ANN has been studied to check if it is possible to predict the damage only with natural frequency obtained from dynamic tests. The network was trained and the result is presented in Figure 6. From the figure, it is observed that each set of the predicted damage is closer to the expected value.

The input test data for the damage levels of $40 \%$ and $70 \%$ were interpolated using ORIGIN software. From that observation, the minimum and the maximum deviations of the various cases and average predicted damage levels along with number of epochs and the performance accuracy of the

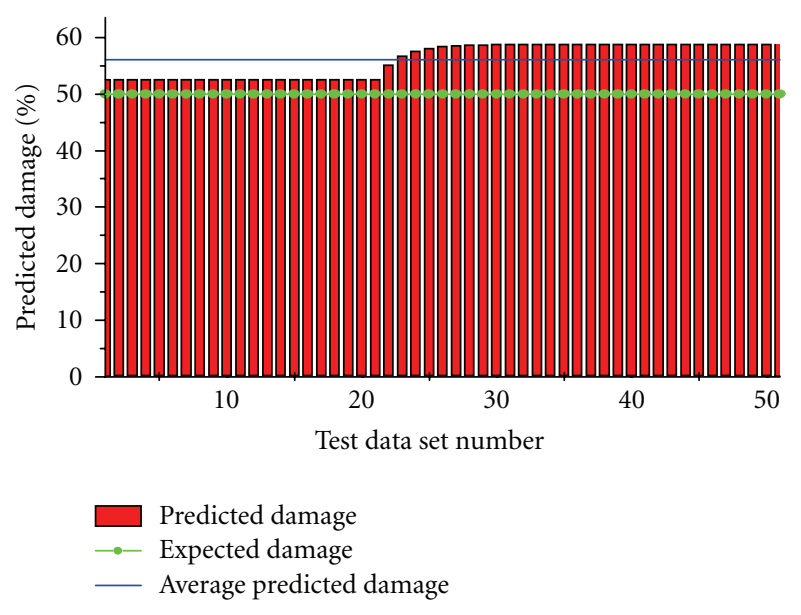

FIGURE 6: Input data set number versus damage predicted by ANN (Case iii).

network are summarised in Table 3. The performance accuracy (user defined value) indicates how close the predicted output of the network is to the expected or targeted output. In our case, the targeted output is the expected damage. The performance accuracy is evaluated from the mean squared error where the error is the difference between the output of the network and the targeted output for a set of inputs.

Epochs were not chosen for these cases, but only the performance accuracy was user defined. So when the accuracy level is obtained as expected, the training process stops. For making comparison, the number of epochs is mentioned. When the network was provided with more number of inputs, the prediction was better in terms of performance accuracy (user defined) and with fewer epochs. The performance accuracy becomes less with more number of epochs required, when the number of inputs starts decreasing. Our aim is to develop the procedure only with natural frequencies and stiffness from data obtained from the field. Therefore, only that particular network was used for developing the procedure.

The table indicates that for all cases the error in the predicted damage can be very large when just one test input data obtained for a single applied load is used to predict the damage level. Maximum error occurred when the ANN was trained with only frequency obtained from the dynamic test as input to the network. This is justified by the fact that the ANN can learn better and predict better when it is trained with more database. This error is still comparable with the errors with which the other ANNs could predict the damage levels. It is also found that the ANN trained with stiffness and frequency obtained over an applied load range within its service load limit could predict the damage very closely (with a maximum difference of $5 \%$ damage) thus paving the way for the development of a new practical method of damage assessment of prestressed concrete beams.

\section{Damage Assessment Procedure}

It has been demonstrated that the ANN trained with natural frequency and stiffness can estimate the damage when tested 


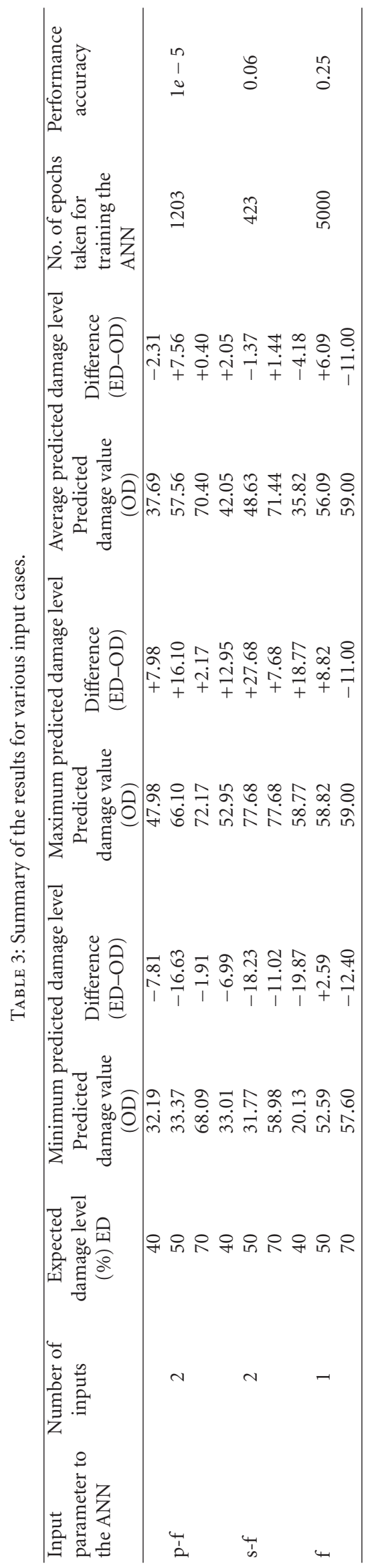


with the data obtained in situ. Also, it has been proved that the ANN trained just with natural frequency can also assess the damage. Based on this concept, a damage assessment procedure has been evolved and presented here. The steps that should be followed for the damage assessment are explained below.

(1) Evaluate the stiffness of the field beam, which is at the postcracking-preyielding stage (service stage) by conducting a static test on the beam. A fraction of the service load is applied for this purpose.

(2) Measure the in situ natural frequency of the field beam by conducting a dynamic test (free vibration test) on the structure.

(3) Evaluate the stiffness ratio, $\left(k / k_{0}\right)$, as the ratio of the measured stiffness of the field beam to the evaluated stiffness of the reference beam in the postcrackingpreyielding stage.

(4) Evaluate the frequency ratio, $\left(f / f_{0}\right)$, as the ratio of the measured frequency of the field beam to the evaluated frequency of the reference beam.

(5) Normalised stiffnesses and the natural frequencies are considered as input, and they are modified using a ratio based on moment of inertia, mass, and length of the field beam. Since the network is trained for a particular cross-section in this study, a ratio is arrived between the sectional properties (moment of inertia, mass, and length) of the field beam to that of the theoretical beam (beam considered in this study) [15]. The normalised stiffness and the natural frequency of the field beam are multiplied by the ratio, and the modified stiffness and the natural frequency are given as the test data in the already trained network.

(6) ANN trained with only natural frequency also can be used in case if it is possible to carry out only free vibration test on the field beam.

(7) The output of the network gives the extent of damage suffered by the field beam.

6.1. Validation of the Damage Assessment Procedure. The above-mentioned procedure is validated using the experimental data presented by Ambrosini et al. [13]. It could be well understood that the requirements of any validation procedure will rarely be available directly in the literature especially dealing with experiments.

Ambrosini et al. [13], National University of Tucumán, Argentina, had conducted static as well as dynamic tests with simply supported conditions on laboratory PSC beam with an I-section of $400 \mathrm{~mm} \times 500 \mathrm{~mm}$ with a test span of $13.30 \mathrm{~m}$. The beam had 20 prestressing bars. To simulate reinforcement damage due to corrosion, the bars were cut, two by two, until reducing the prestressing reinforcement to a $50 \%$. The beam was subjected to two-point loads at onethird points. The dynamic measurement system composed of acceleration transducers, a dynamic amplifier, and data
TABLE 4: Comparison of predicted values with Ambrosini et al. [11].

\begin{tabular}{lcc}
\hline Serial no. & \multicolumn{2}{c}{ Degree of damage (\%) } \\
& $\begin{array}{c}\text { Expected degree of damage } \\
\text { (Ambrosini et al.) }\end{array}$ & Predicted damage \\
\hline 1 & 30 & 29.93 \\
2 & 40 & 41.61 \\
3 & 50 & 51.09 \\
\hline
\end{tabular}

acquisition equipment consistent in a computer with a differential eight-channel card. Dynamic tests were performed by exciting the beam with a hammer blow in the central section. The natural frequencies obtained in each one of the test stages are tabulated and presented in the literature. These frequencies were normalized and modified based on the ratio of sectional properties since the training is done for a beam, which has different section. The modified frequencies are fed as test input to the trained network, and the prediction of ANN gives the degree of damage. The ANN trained only with natural frequencies has been used here to validate the ANNbased theoretical approach for damage assessment since the stiffness data were not available in the literature. Table 4 explains how the damage assessment procedure developed in his study works. From Table 4, it is demonstrated that the ANN approach can predict the damage very closely.

\section{Conclusions}

A damage assessment procedure based on ANN for the prestressed concrete rectangular beams has been formulated, and the procedure has been validated using the data available from the literature. From this study, it can be concluded that

(i) an ANN trained with dynamic data obtained at different loads of a prestressed concrete beam is sufficient to assess the damage level. An ANN trained with a mix of static and dynamic data can be used to confirm the prediction of an ANN trained with dynamic data, if needed. The average of the predicted damage levels for test data obtained at different loads is the best method to assess the damage by ANN;

(ii) an assessment technique leading to the quantitative evaluation of degree of damage is possible by ANN using the natural frequency and stiffness in the postcracking-preyielding range as input data;

(iii) ANN can be used as an effective tool in the damage assessment of prestressed concrete beams;

(iv) damage assessment procedure developed in this research work can effectively be put in to use for damage assessment of field beams.

\section{References}

[1] M. M. Abdel Wahab and G. Roeck, "Damage detection in bridges using modal curvatures: application to a real damage scenario," Journal of Sound and Vibration, vol. 226, no. 2, pp. 217-235, 1999. 
[2] Z. Zhengjie, D. W. Leon, F. S. Bruce et al., "Structural health monitoring of precast concrete box girders using selected vibration-based damage detection methods," Advances in Civil Engineering, vol. 2010, Article ID 280685, 21 pages, 2010.

[3] P. Cawley and R. D. Adams, "The location of defects in structures from measurements of natural frequencies," Journal of Strain Analysis, vol. 14, no. 2, pp. 49-57, 1979.

[4] H. Abdul Razak and F. C. Choi, "The effect of corrosion on the natural frequency and modal damping of reinforced concrete beams," Engineering Structures, vol. 23, no. 9, pp. 1126-1133, 2001.

[5] A. Carpinteri, S. Invernizzi, and G. Lacidogna, "In situ damage assessment and nonlinear modelling of a historical masonry tower," Engineering Structures, vol. 27, no. 3, pp. 387-395, 2005.

[6] X. Wu, J. Ghaboussi, and J. H. Garrett, "Use of neural networks in detection of structural damage," Computers and Structures, vol. 42, no. 4, pp. 649-659, 1992.

[7] Q. Chen, Y. W. Chan, and K. Worden, "Structural fault diagnosis and isolation using neural networks based on responseonly data," Computers and Structures, vol. 81, no. 22-23, pp. 2165-2172, 2003.

[8] X. Fang, H. Luo, and J. Tang, "Structural damage detection using neural network with learning rate improvement," Computers and Structures, vol. 83, no. 25-26, pp. 2150-2161, 2005.

[9] J. L. Zapico, K. Worden, and F. J. Molina, "Vibration-based damage assessment in steel frames using neural networks," Smart Materials and Structures, vol. 10, no. 3, pp. 553-559, 2001.

[10] K. V. Yuen and H. F. Lam, "On the complexity of artificial neural networks for smart structures monitoring," Engineering Structures, vol. 28, no. 7, pp. 977-984, 2006.

[11] N. Bakhary, H. Hao, and A. J. Deeks, "Damage detection using artificial neural network with consideration of uncertainties," Engineering Structures, vol. 29, no. 11, pp. 2806-2815, 2007.

[12] O. R. de Lautour and P. Omenzetter, "Prediction of seismicinduced structural damage using artificial neural networks," Engineering Structures, vol. 31, no. 2, pp. 600-606, 2009.

[13] D. Ambrosini, B Luccioni, and R. Danesi, "Theoretical-experimental determination in prestressed concrete beams," NDT Net, vol. 5, no. 7, pp. 1-5, 2000.

[14] C. Antony Jeyasehar and K. Sumangala, "Nondestructive evaluation of prestressed concrete beams using an artificial neural network (ANN) approach," Structural Health Monitoring, vol. 5, no. 4, pp. 313-323, 2006.

[15] K. Sumangala, Damage assessment and rehabilitation of prestressed concrete rectangular beams, Ph.D. thesis, Annamalai University, Tamilnadu, India, 2005. 

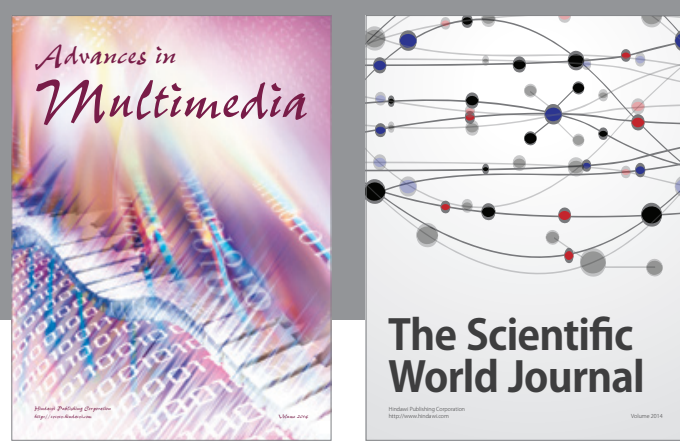

The Scientific World Journal
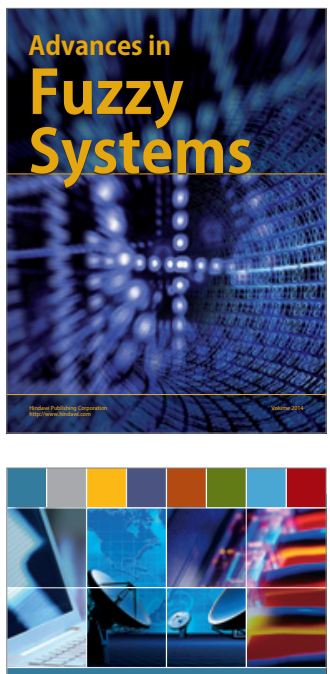

Computer Networks and Communications
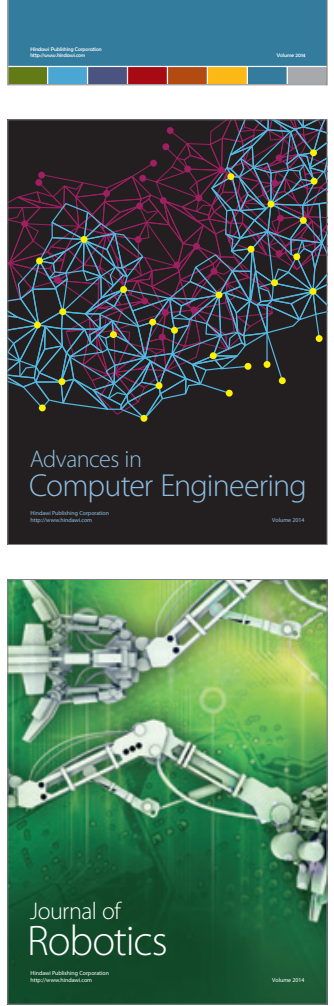
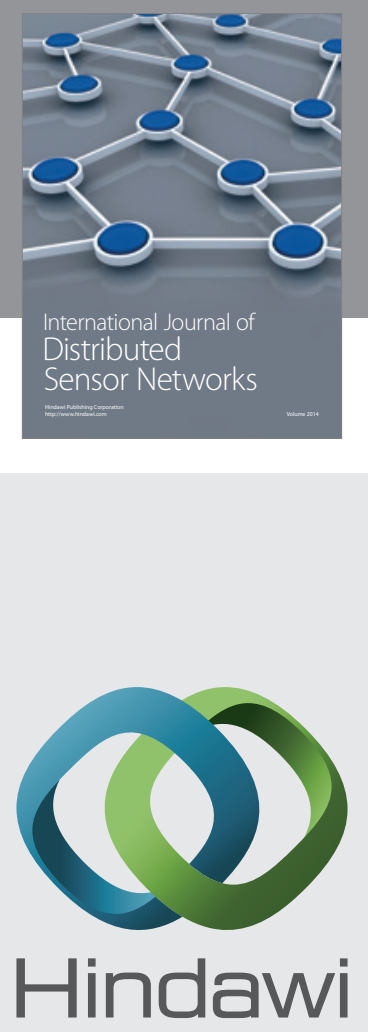

Submit your manuscripts at

http://www.hindawi.com
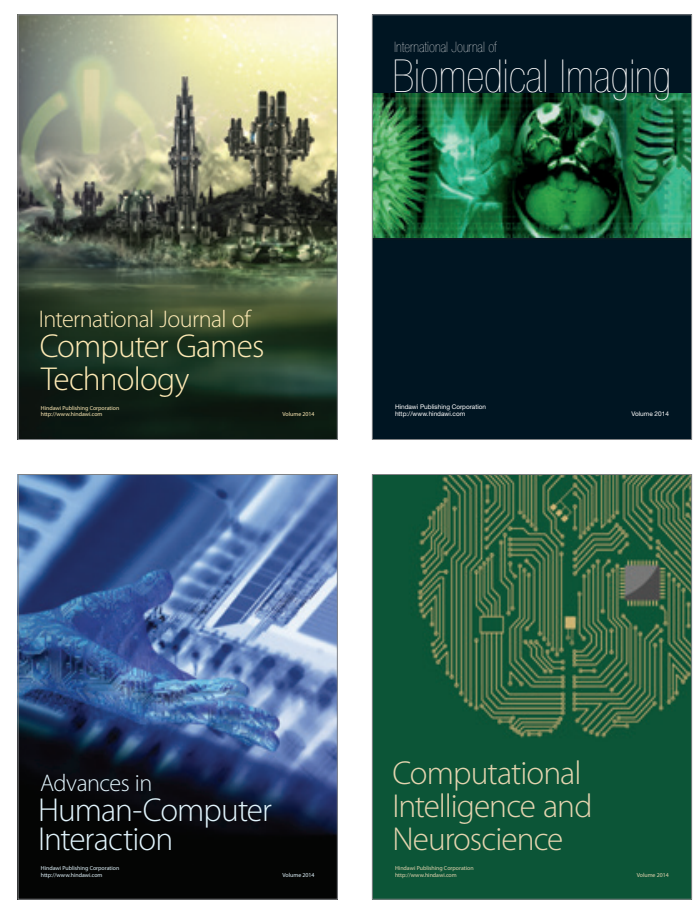
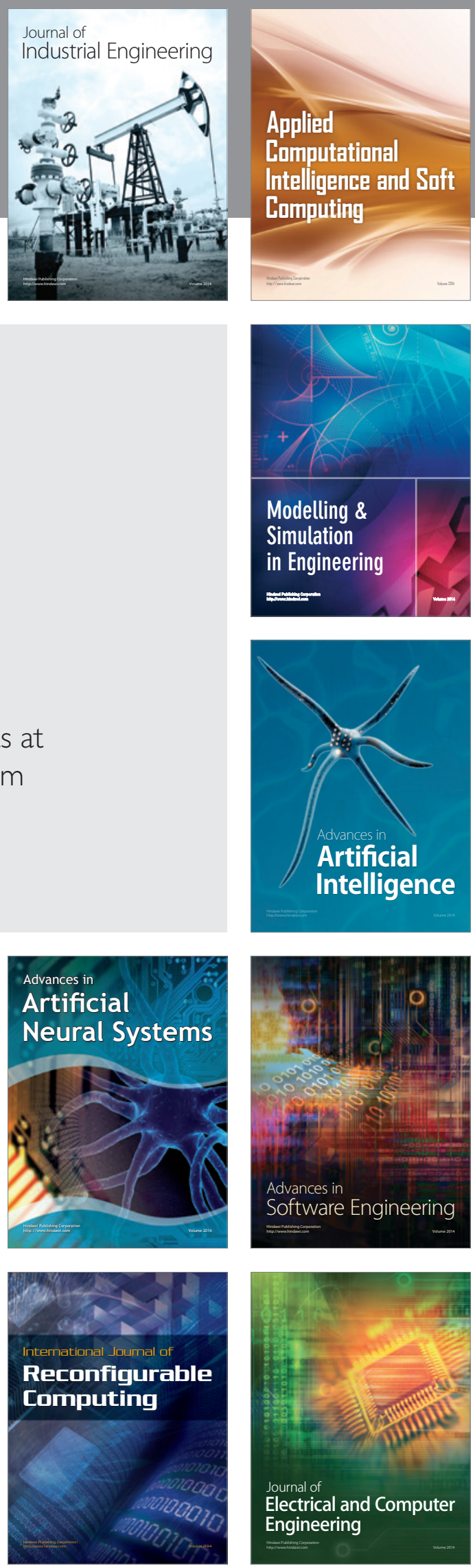